\title{
Elementos para un concepto sociológico de innovación
}

\author{
Elements for a sociological concept of innovation \\ Holm-Detlev Köhler y Sergio González Begega \\ Universidad de Oviedo \\ hkohler@uniovi.es /gonzalezsergio@uniovi.es (ESPAÑA)
}

Recibido: 15.10 .2013

Aceptado: 28.05 .2014

\section{RESUMEN}

El término innovación ha adquirido un carácter casi omnipresente en el contexto de la intensificación competitiva que acompaña a la globalización. El fomento de la capacidad innovadora figura entre los principales objetivos de los gestores públicos y privados. Sin embargo, se carece de un concepto académico debidamente elaborado de innovación, más allá de los enunciados genéricos y estrechos comúnmente manejados en los ámbitos económicos y de negocio. El artículo explora un conjunto de fuentes que podrían utilizarse para elaborar un concepto sociológico de innovación susceptible de ser incorporado a las actuales teorías sobre organización económica y cambio institucional. Para ello, se revisan tres corrientes de reflexión teórica sobre innovación, que se integran en un mismo paradigma de la innovación: las aportaciones de la Economía Política de Marx y Schumpeter; las contribuciones de la Economía Evolutiva; y la Economía Política del Desarrollo. A partir de ellas, se propone una re-conceptualización sociológica de la idea de innovación ajustada a la teoría socioeconómica de instituciones dinámicas.

\section{PALABRAS CLAVE}

Innovación, conocimiento, competitividad, cambio institucional, sociología económica.. 


\begin{abstract}
Innovation is an almost omnipresent term in the current context of globalization and intense competition. Increased innovative capacity figures among the main objectives of governments and managers. However, we still lack an elaborated academic concept of innovation facing its all too generic and narrow use in the economic and business discourse. This article argues for a sociological concept of innovation to incorporate it in the modern social theories on economic organizations and institutional change. This implies its systematic linking with concepts like knowledge, competitiveness and competence. To this end we analyze the three most relevant streams of innovation theory that can be integrated in a new innovation paradigm: the political economy from Marx to Schumpeter, evolutionary economics and the political economy of development. Then we propose a sociological reconceptualization of the notion of innovation in the context of a socio-economic theory of dynamic institutions.
\end{abstract}

\title{
KEY WORDS
}

Innovation, knowledge, competitiveness, institutional change, economic sociology.

\section{INTRODUCCIÓN ${ }^{1}$}

"We owe it all to the hippies" (Brand 1995).

A mediados de la década de los setenta, dos jóvenes norteamericanos, Steven Jobs y Steven Wozniak, ensamblaban y comercializaban su Apple I mientras IBM invertía millones de dólares en Investigación y Desarrollo (I+D). Por las mismas fechas, Amancio Ortega e Ingvar Kamprad, fundadores de Inditex (Zara) e IKEA, exploraban los primeros trechos de sus exitosas estrategias de negocio, también fundamentadas en la innovación, aunque en un sentido bien distinto: en sectores de baja intensidad tecnológica, sin patentes técnicas y sin departamento de I+D.

La existencia de brechas tecnológicas entre sectores productivos plantea un primer reto para cualquier reflexión teórica sobre la innovación. ¿Es posible y, de serlo, cómo - elaborar un concepto de innovación capaz de aprehender la extraordinaria diversidad de la actividad innovadora y de los tipos de innovación, radical, modular e incremental? El problema, además, no solo se plantea entre sectores productivos sino también entre organizaciones que compiten en una misma área de actividad. Como han comprobado los competidores de

1 El artículo se apoya en los resultados del proyecto PILOT, Policy and Innovation in Low Tech. Knowledge, Employment and Growth Contribution of the Old Economy Industries in Europe (http://www.pilot-project.org), financiado por el Sexto Programa Marco de Investigación y Desarrollo Tecnológico de la Comisión Europea, (referencia UE-02-HPSE_CT_000112). 
Apple, Inditex o IKEA, no resulta sencillo reducir la distancia de competitividad emulando las soluciones organizativas de estas empresas o reclutando a sus talentos directivos. La fuerza competitiva que deriva de la innovación no reside únicamente en los conocimientos individuales sino más bien en su concentración y combinación eficaz en forma de conocimientos colectivos.

La capacidad innovadora de una organización es universalmente reconocida como una fuente de ventaja competitiva. Sin embargo, se carece de un concepto teórico de innovación desarrollado. De hecho, "en la retórica más habitual de la innovación se revela una falta de comprensión de lo que esta significa: una creación imprevisible, más bien escasa y siempre social" (Innerarity 2009: 30).

La intensificación competitiva que acompaña a la globalización ha convertido al término innovación en un mantra, "una especie de remedio que todo lo cura, el bálsamo de Fierabrás contemporáneo" (Castro Martínez y Fernández de Lucio 2013: 22), bajo el imperativo simple «de innova o muere» (Cooper 2005: 4). Tras la Cumbre de Lisboa, la Unión Europea ha adquirido el objetivo de convertirse en una economía competitiva basada en el conocimiento y la innovación, como respuesta a los retos de la globalización (Comisión Europea 2007) o, más recientemente, de la crisis financiera (Comisión Europea 2013).

La innovación es reconocida asimismo como el factor clave que asegura la capacidad competitiva sostenible de organizaciones y territorios, frente a los conocimientos codificados y los recursos materiales, de mayor disponibilidad. Sin embargo, y pese al uso inflacionario del término, la innovación, concebida como el resultado de un proceso social, ha ocupado una posición marginal en el espacio académico.

Los modelos económicos neoclásicos no han dado cabida al sentido social de la innovación. Las teorías de administración de empresa, por su parte, se han limitado a desarrollar conceptos normativos de innovación, construidos únicamente a partir de la identificación de los estilos de gestión y de difusión. La Sociología tampoco ha podido (o ha tenido interés en) captar la compleja interrelación de la innovación con los entornos socio-culturales y organizacionales. Buena parte de los trabajos sociológicos sobre innovación han restringido sus preocupaciones al análisis del impacto de la innovación tecnológica sobre los usos sociales de las Tecnologías de la Información y de la Comunicación (TICs), dejando el análisis sistemático de la fisiología de la innovación a otras disciplinas. Así, resulta paradójico que "quienes se ocupan de la innovación están poco interesados en la sociedad y quienes piensan en la sociedad no parecen haber entendido la centralidad que la innovación tiene a la hora de comprender nuestras sociedades" (Innerarity 2009: 19).

Fageberg y Verspagen (2009) confirman el papel marginal de la Sociología frente a ciertos enfoques económicos heterodoxos, como la Economía Evolutiva post-schumpeteriana y otras propuestas neo-institucionalistas, en el desarrollo de la innovación como nuevo espacio de reflexión científica. El único sociólogo clásico citado habitualmente por la literatura sobre innovación es Karl Marx y la única aportación estrictamente sociológica relevante dentro 
de esta misma literatura es la Teoría de Difusión de Innovación, que emana de la obra pionera de Everett Rogers (1962). En ella, la innovación queda definida como algo subjetivo, una idea percibida como nueva por una comunidad de individuos y difundida mediante diferentes canales y redes de comunicación (Rogers 1962: 11). Sin embargo, la Teoría de la Difusión de Innovación no aborda el problema de la génesis de la innovación dentro de un contexto estructurado y a través de la acción social.

El artículo discute las posibilidades de desarrollo de un concepto sociológico de innovación que cubra este vacío y que pueda incorporarse a las teorías actuales sobre organización económica y cambio institucional. La estructura del artículo será la siguiente: en primer lugar, se efectuará una revisión de las limitaciones del enfoque de la Economía neo-clásica sobre innovación para, a continuación, examinar las aportaciones de otras perspectivas económicas heterodoxas que elaboran construcciones teóricas en las que la acción social toma presencia explícita o implícita. La Economía Política de Marx y de Schumpeter, la Economía Evolutiva y la Economía Política de Desarrollo han contribuido a la conformación de un paradigma post-schumpeteriano y neoinstitucional de innovación que es apropiado para fundamentar un concepto sociológicamente construido de innovación. La segunda sección del artículo abordará las posibilidades de adaptación de este utillaje teórico para una reelaboración sociológica del concepto de innovación, revisando las fuentes sociales del proceso innovador desde la perspectiva del trabajo, de cambio socio-institucional y de las organizaciones. Por último, el artículo recoge unas conclusiones en las que, además de una recapitulación crítica de contenidos, se enumera un conjunto de dificultades analíticas inherentes a cualquier esfuerzo de teorización sobre los procesos de innovación.

\section{UNA PERSPECTIVA OLVIDADA. LA INNOVACIÓN COMO PRO- DUCTO SOCIAL.}

La innovación resulta de la acción social, constituye un proceso interactivo entre actores orientado a la generación de nuevos conocimientos. Como producto social, la innovación no encuentra acomodo en los modelos de la Economía neoclásica. En su entramado de agentes racionales, acceso ilimitado a la información y equilibrio perfecto, los mecanismos que explican el proceso de la actividad innovadora permanecen sistemáticamente ocultos. La existencia de carencias teóricas, no obstante, no parece haber sensibilizado a una comunidad académica poco preocupada, en realidad, por el significado social de la innovación, por su relevancia y por el análisis de su evidencia empírica. El origen de este enfoque, acrítico y recursivo, se encuentra en el informe "Science. The Endless Frontier", elaborado por la Oficina de Investigación Científica y de Desarrollo de Estados Unidos bajo iniciativa de su director, Vannevar Bush (1945). En este documento se establece la interpretación ortodoxa de la secuencia de innovación, a partir de un modelo lineal de cascada o carrera de relevos según el cual la 
innovación se despliega a lo largo de una cadena necesaria que arranca en la investigación científica básica, se transfiere a la investigación aplicada y resulta en un nuevo producto que, una vez testado, es introducido en el mercado para su comercialización (Castro y Fernández de Lucio 2013: 73). La influencia de este modelo es tal que incluso la Teoría de Difusión de Innovación de Rogers (1962) se acomoda a esta perspectiva lineal que comienza en el científico concebido como innovador y desemboca en el mercado. Los modelos de la gestión de innovación en la literatura gerencial y de administración de empresas mantienen esta construcción lineal y secuencial del proceso de innovación, ofreciendo herramientas para gestionarla en sus distintas fases (Eveleens 2010).

Los problemas de este enfoque, ahistórico y estático, se hicieron evidentes cuando los departamentos académicos de Administración de Empresas decidieron incorporar a su agenda de investigación la cuestión de la innovación y se propusieron identificar las variables explicativas de los diseños efectivos y no efectivos de las políticas de innovación. Los modelos de la Economía neoclásica se vieron entonces desbordados, debido a su incapacidad para explorar el dinamismo social inherente a cualquier proceso de innovación y sus densas interconexiones con los procesos de cambio institucional. La apertura de una tensión entre los útiles teóricos disponibles y la existencia de creciente evidencia empírica sobre innovación que demandaba el desarrollo de instrumentos más apropiados para su interpretación abrió la puerta a nuevos nichos de pensamiento económico heterodoxo, que subrayaron la necesidad de abordar el problema de la innovación desde presupuestos teóricos distintos. Ni la innovación ni los procesos de aprendizaje estratégico de las organizaciones sobre ésta pueden entenderse como el resultado de un conjunto de decisiones individuales, racionales y ordenadas. Al contrario, la innovación debe estudiarse como un proceso social, como el producto de la movilización de competencias colectivas y conocimientos compartidos no reducibles a un modelo mecánico y racional.

\section{LAS APORTACIONES DE LA ECONOMÍA POLÍTICA. DE SCHUMPETER A MARX.}

El reconocimiento de la complejidad social de la innovación alimentó la aparición de nuevas piezas teóricas que, desde intersticios entre disciplinas académicas, insistieron en el carácter endógeno y socialmente condicionado de la innovación. La innovación es un proceso social que tiene su origen en el interior de la organización (Larrea 2010; Mytelka y Smith 2002).

Paradójicamente, las primeras dudas sobre la validez de los modelos neoclásicos para el análisis de la innovación se encuentran en uno de los principales exponentes de la ortodoxia económica neo-clásica del siglo XX: Friedrich A. Hayek.

"Si contamos con toda la información relevante, si podemos partir de un sistema de preferencias predeterminado y si tenemos el conocimiento completo de los medios disponibles, el problema resultante es de pura lógica (...). Sin 
embargo, esto enfáticamente no es el problema económico que la sociedad enfrenta. El carácter peculiar de un orden económico racional está determinado precisamente por el hecho de que el conocimiento de las circunstancias de las que tenemos que hacer uso nunca existe en forma concentrada o integrada, sino únicamente como fracciones dispersas de conocimiento incompleto $\mathrm{y}$ frecuentemente contradictorio que todos los individuos poseen de forma separada" (Hayek 1945: 519) (traducción propia).

Hayek subraya así la necesidad de abordar la movilización de los conocimientos como un entramado disperso, que no reside en individuos aislados y perfectamente informados sino que responde a la interacción cooperativa de múltiples actores sociales cuyas decisiones pueden no resultar de cálculos económico-racionales.

Ahora bien, el primer economista verdaderamente iconoclasta que abandona la modelización neo-clásica de la innovación es Joseph A. Schumpeter. Para este autor, la innovación científica básica no es necesariamente el principio del camino y, además, tiene un papel secundario frente a la innovación aplicada que se lleva a cabo en el interior de las organizaciones económicas. Decisivamente, el principal agente de innovación se encuentra en el interior de la empresa y no fuera de ella, a través de la figura del innovador individual heroico (Schumpeter 1911) o de las estructuras burocratizadas de I+D de las grandes corporaciones industriales (Schumpeter 1942).

Como señala Elliott (1980), Schumpeter aboga por una visión institucionalista del capitalismo como sistema dinámico de destrucción creativa y dialéctica entre las fuerzas productivas y concibe la gran empresa industrial como el motor del cambio en su búsqueda de ventajas monopolísticas dentro del mercado. Para Schumpeter, el equilibrio del modelo de competencia perfecta neo-clásico no solo es completamente excepcional sino que, de prolongarse en el tiempo, constituiría un freno al desarrollo económico. Las empresas individuales invierten en la generación de nuevos conocimientos y persiguen la innovación para alcanzar posiciones de competencia monopolista sobre el mercado y es, precisamente, la adquisición de estas ventajas competitivas discontinuas que se apoyan sobre la innovación, la que sacude a la economía capitalista del letargo. Así, la dinámica del mercado no es resultado de la competencia de precios entre productos y productores homogéneos sino de su diferenciación en calidades.

"El impulso fundamental que pone y mantiene en movimiento a la máquina capitalista procede de los nuevos bienes de consumo, de los nuevos métodos de producción y transporte, de los nuevos mercados, de las nuevas formas de organización industrial que crea la empresa capitalista" (Schumpeter 1942: 120).

La ruptura con el modelo neo-clásico de innovación se completa si, a la crítica de Schumpeter, se le suma la visión marxiana del factor trabajo como fuente de innovación (Denis, 2012). La innovación no resulta únicamente de la caza de beneficios monopolistas por parte de la empresa o de la institucionalización de la I+D en las grandes corporaciones sino también, y decisivamente, de la interacción entre los distintos actores del proceso de generación de valor. La dinámica de innovación se despliega a lo largo de ese 
proceso y se entrecruza con las relaciones sociales de producción. La innovación no sigue la lógica de la racionalidad técnica o económica sino que refleja las constelaciones de poder existentes en la organización, fracturadas más allá de la tradicional división entre capital y trabajo (Köhler 2007; MacKenzie 1984).

La innovación tiene su origen en el proceso de trabajo y en la valoración del mismo. Por muy importantes que sean las aportaciones del innovador heroico, el círculo de la actividad innovadora no se agota en el individuo sino que se extiende al trabajo humano colectivo. La innovación descansa sobre la combinación de factores productivos en el proceso de creación de valor, a partir de dos funciones sociales básicas: (1) la empresarial o de puesta en valor y cosificación del trabajo vivo; y (2) la del trabajo, como vehículo de la actividad productiva y fuente del valor añadido. El proceso organizado de puesta en valor del trabajo se convierte en la fuente social de toda actividad de innovación.

"Las mejoras constantes que pueden conseguirse (...) responden única y exclusivamente a las experiencias y observaciones sociales que permite la producción del obrero colectivo combinado a gran escala (...). Solo la experiencia del obrero combinado descubre y revela dónde y cómo puede economizarse, cómo pueden aplicarse del modo más sencillo los descubrimientos ya realizados, qué fricciones prácticas hay que vencer para aplicar la teoría, llevándola a la práctica en el proceso de la producción" (Marx 1897: 93, 95, 115).

\section{EL CONCEPTO DE INNOVACIÓN EN LA ECONOMÍA EVOLUTIVA.}

La perspectiva analítica de la innovación que plantea la Economía Evolutiva se apoya en las propuestas de Schumpeter que, según Nelson (1994: 111) "hay que entender como una teoría explicativa de los procesos de aprendizaje en la economía y en la sociedad" (traducción propia). La innovación constituye así un elemento clave para la comprensión de la competencia en la economía post-industrial, revistiéndose de un carácter contingente y desvinculándose de cualquier noción de la evolución económica darwinista o racional-optimizadora. La Economía Evolutiva subraya que el criterio de selección de mejoras implícito en todo proceso de innovación no es necesariamente la superioridad de las soluciones científica o técnicamente más fuertes sino el resultado de tensiones entre intereses contradictorios y luchas de poder entre actores (Loasby 2002).

La noción de innovación de la Economía Evolutiva atiende también a algunos problemas microeconómicos recurrentes que el modelo interpretativo neo-clásico se muestra incapaz de solventar. La Economía neo-clásica no da cabida a la empresa como forma no mercantil de gestión de recursos, que se encuentran irremediablemente sujetos a la compra en el mercado. Desde su perspectiva, la empresa es un actor atrapado entre las leyes de la oferta y la demanda, una simple 'caja negra' inerte (Rosenberg, 1993). Los primeros intentos de romper esta inercia y abrir la caja negra de la toma de decisiones 
empresariales llevan a Ronald Coase (1937) a formular la Teoría de los Costes de Transacción, que posteriormente desarrolla Oliver Williamson $(1981 ; 1985)$. En condiciones de información limitada, los actores pueden encontrar más económico crear y conservar el conocimiento propio que intentar adquirirlo en el mercado. No obstante, la innovación surge de motivaciones más complejas que la contención de los costes de transacción asociados a la adquisición de conocimiento.

La Economía Evolutiva se apoya en el enfoque de la Empresa como Conjunto de Recursos de Edith Penrose (1959) para explicar las fuentes de la innovación. La empresa constituye un complejo de conocimientos de producción acumulados que facilitan y orientan la generación y aplicación de nuevos conocimientos. La mutualización de capacidades convierte a la empresa en un actor social dinámico de generación de conocimiento (Pavitt 1992). Así, el conocimiento resulta de procesos interactivos entre múltiples agentes, a través de la combinación contextual de experiencias teóricas y prácticas, siendo especialmente relevantes para la innovación las que hacen referencia a la dimensión tácita del conocimiento colectivo (Polanyi 1966; Böhle 2010; Lam 2000).

Tabla 1. Tipos de conocimiento según Lundvall (2002); Spender (1996) y Blackler (1995).

\begin{tabular}{|c|c|c|}
\hline & Individual & Colectivo \\
\hline Explícito & $\begin{array}{c}\text { Consciente (Spender), interiorizado } \\
\text { (Blackler) o Know-what (Lundvall) }\end{array}$ & $\begin{array}{c}\text { Objetivado (Spender), } \\
\text { codificado (Blackler) o } \\
\text { Know-why (Lundvall) }\end{array}$ \\
\hline Tácito & $\begin{array}{c}\text { Automático (Spender), incorporado } \\
\text { (Blackler) o Know-how (Lundvall) }\end{array}$ & $\begin{array}{c}\text { Colectivo (Spender), } \\
\text { digerido o incorporado } \\
\text { a la cultura (Blackler) o } \\
\text { Know-who (Lundvall) }\end{array}$ \\
\hline
\end{tabular}

Fuente: Adaptado de Serra Ramoneda 2001: 799

La ventaja sistemática de la empresa frente al mercado reside en la dimensión colectiva y tácita del conocimiento. Es ahí donde la empresa encuentra la fuente de ventaja competitiva sostenible, aunque la innovación trascienda los diferentes tipos de conocimiento, codificados y tácitos, individuales y colectivos. Por eso la función esencial de la empresa no es la adquisición de conocimientos explícitos y codificados (si así fuese, el éxito innovador respondería a un problema de vigor financiero) sino la generación, atesoramiento y transmisión de conocimientos tácitos que no se pueden adquirir en el mercado y que son difícilmente reproducibles por los competidores.

"El conocimiento codificado es genérico y no condicionado por un tiempo, lugar o grupo de personas particulares. Las reglas, las regulaciones o los manua- 
les de operaciones estandarizadas son buenos ejemplos de ello. El conocimiento tácito, en cambio, es dinámico, desarrollado y transmitido informalmente e inevitablemente inmerso en la experiencia colectiva de un determinado grupo de trabajo o colectivo ocupacional" (McKinlay 2005: 243) (traducción propia).

Hirsch-Kreinsen (2002) indica que la dimensión intangible y tácita del conocimiento práctico, en contraposición al teórico, entrelaza elementos individuales y colectivos, es informal, no está codificada y se encuentra inmersa en las rutinas de comunicación y cooperación. El conocimiento práctico estructura el sistema social de una comunidad de conocimiento, sea esta una organización, una red o una región. Desde esta perspectiva, las empresas son organizaciones de aprendizaje incrustadas, que forman racimos o clusters de innovación con otras empresas, entidades de I+D y actores políticos, dentro del territorio en el que se asientan (Dosi et al. 1992).

La Teoría Evolutiva de la Empresa construye una relación compleja entre rutinas organizacionales, procesos de búsqueda de nuevos conocimientos y entornos selectivos. Las primeras alojan los conocimientos operativos disponibles por la organización. Los segundos engloban las actividades de mejora o reemplazo de los mismos. Los terceros aportan los impulsos externos y las restricciones que orientan los procesos de búsqueda de conocimientos. El principal problema al que se enfrenta la acción social empresarial a este respecto es la generación de una dinámica equilibrada entre rutinas y procesos creativos. Esta dinámica refleja la competencia sintetizadora de la empresa, incluyendo sus propiedades dinámicas, de absorción y arquitectónicas, es decir, su capacidad para incorporar conocimientos externos, transformarlos y combinarlos de manera estratégica con sus competencias internas (Bender 2003).

Al concebir la empresa como organización de aprendizaje basada en el conocimiento, otra de las aportaciones de este enfoque es el desdibujado de las fronteras entre trabajo cognitivo y manual y entre funciones innovadoras y rutinarias. Así, la Economía Evolutiva rebasa la idea schumpeteriana de innovación, que no cuestionaba esta asociación. Para la Economía Evolutiva, la innovación traspasa los límites de los departamentos de I+D y se incorpora a todas las actividades estratégicas y operativas de la empresa. La permeabilidad de los distintos tipos de trabajo a la innovación incrusta a la organización en sus entornos territoriales y sectoriales.

La Economía Evolutiva reconoce la existencia de una relación de interdependencia entre la empresa y el entorno que condiciona la capacidad de innovación. Bender (2006) formula el concepto de capacidades de fomento de la innovación y distingue en él dos dimensiones analíticas sobre capacidades: (1) las capacidades transformativas de la organización, que aluden a la transformación de conocimientos en competencias operativas organizacionales; y (2) las capacidades configuracionales, que indican la generación interna de nuevos conocimientos o combinaciones de recursos internos a través de la integración de conocimientos dispersos. La innovación, así concebida, engloba la "sintetización" de conocimientos dispersos, la "organización" en forma del entrelazado de agentes competentes dispersos y el "diseño" o articulación creativa de resultados 
de innovación (Henderson y Clark 1990; Teece, Pisano y Shuen 1997). Se trata de una capacidad compleja de innovación que permite asociar, "crear y sostener una arquitectura robusta de generación y uso de conocimientos desde una amplia variedad de fuentes entre los que se encuentran los empleados, los proveedores, los clientes y los organismos públicos" (Cooke y Morgan 1988: 17) (traducción propia).

\section{LA ECONOMÍA POLÍTICA DEL DESARROLLO: LOS SISTEMAS DE INNOVACIÓN}

La comprensión de los procesos de innovación lleva necesariamente al análisis de la incrustación de la empresa en su entorno. La innovación forma parte de redes interactivas y racimos de innovación en los que la empresa se relaciona con otras organizaciones e instituciones. La incrustación institucional de la empresa constituye el tercer pilar del paradigma de innovación postschumpeteriano.

La Economía Política del Desarrollo ha investigado de forma intensiva la cooperación empresarial en red y el desarrollo compartido de conocimientos como elemento explicativo de la elevada competitividad y capacidad de innovación de algunas regiones de alto éxito económico. Esta perspectiva ha descubierto que solo una pequeña parte del conocimiento científico básico es transferido a las tecnologías aplicadas y que, en realidad, el vínculo entre innovación científica básica e innovación aplicada es más tenue de lo que cabría esperar. De hecho, es la actividad innovadora aplicada de las empresas (y sobre todo de las PYMES) la que, a partir de la apropiabilidad del conocimiento tácito, resulta crítica para explicar el éxito económico de las regiones (Lundvall 2002).

Tal y como indican Lundvall et al. (2002) o Freeman (2002), esta intuición sobre el carácter institucionalmente incrustado del conocimiento ya estaba presente en la crítica que Friedrich List (1841) hace de las abstracciones ahistóricas y aempíricas en las que incurren los modelos económicos clásicos al enunciar que son las leyes generales del mercado las que determinan el grado de desarrollo de una economía nacional. List incide en que no son éstas sino las instituciones las que desempeñan un papel fundamental.

La importancia de las redes institucionales para el fomento de la innovación, en forma de sistemas territoriales de innovación de rango variable (nacional o regional) ha sido explorada por autores como Nelson (1993), Porter (1990) o Freeman (1987). Desde un ámbito distinto, los enfoques de variedades de capitalismo (Hall y Soskice 2001) y de sistemas nacionales de negocio (Whitley 1999) incluyen la innovación entre los dispositivos institucionales que moldean las distintas formas de organización económica. La innovación se entrelaza con el sistema financiero, el educativo o las relaciones laborales, entre otros y retroalimenta al conjunto institucional a través de procesos de aprendizaje cumulativos e interactivos (Werle 2005). A escala regional, la estabilidad y proyección a largo plazo de esta retroalimentación resulta determinante para 
la generación de ventajas competitivas, tal y como señalan los debates sobre distritos industriales y milieus innovadores.

"Los sistemas regionales de innovación son entornos donde una densa comunicación entre empresas, unas estructuras socio-culturales y un entorno institucional estimulan el aprendizaje social y territorialmente integrado y la innovación continua. Este concepto es un oxímoron que expresa dos conceptos antitéticos (...). Si bien la idea de sistema hace hincapié en el papel estable del orden regional, la de innovación enfatiza el proceso de destrucción creativa" (Heidenreich 2004: 367) (traducción propia).

Desde una perspectiva distinta a la de la articulación territorial, otros estudios han evaluado las particularidades sectoriales de los sistemas de innovación, acercándose al conjunto de conocimientos, tecnologías básicas, cadenas de valor y sistemas ocupacionales y de formación de determinadas industrias (Jürgens y Sablowski 2005; Malerba 2004; Pavitt 1984). Así, en sectores como el metalmecánico serían dominante la innovación incremental y de aplicación práctica, mientras que en sectores como la biotecnología o la industria farmacéutica sería más relevante la investigación científica básica llevada a cabo por departamentos de I+D.

Deutschmann (2002) identifica cuatro ciclos de innovación y conocimientos, distintos en importancia, duración e intensidad para cada sector o área de actividad: (1) la génesis o ciclo de innovaciones radicales a partir del desarrollo de nuevos conocimientos básicos; (2) la difusión o ciclo de introducción de modificaciones para la aplicación e imitación masiva de la innovación; (3) la institucionalización o ciclo de institucionalización de la innovación y añadido de innovaciones incrementales; y (4) la crisis o ciclo de burocratización, esclerosis institucional y atrapado de la innovación.

La actividad innovadora discurre entre estos ciclos a través de procesos interactivos protagonizados por diversos actores organizados en red. Precisamente, es este carácter social y reticular de la innovación el que invita a desarrollar un concepto sociológico de la innovación. Este concepto, a cuyos componentes se alude en la siguiente sección, debe construirse sobre un conjunto de certidumbres aportadas por la Economía heterodoxa en su deconstrucción del paradigma neo-clásico: (1) no existe, en realidad, una relación directa entre conocimiento científico e innovación; (2) el conocimiento, concebido como fuente de innovación, lleva implícito un carácter colectivo e institucional con alta densidad de componentes tácitos y no comercializables; (3) la innovación como instrumento de competitividad no tiene su origen en las particularidades tecnológicas de determinados sectores o en el tamaño de las organizaciones, puesto que las industrias de baja intensidad tecnológica y las PYMES también pueden ser innovadoras; (4) la innovación no solo se refiere a productos y procesos sino también a formas de organización y de comunicación; y, por último, (5) la innovación no se ajusta a una secuencia lineal y teleológica sino que responde a constantes acoplamientos recursivos entre los procesos de I+D, de fabricación, de aplicación y de mejoras incrementales. 


\section{HACIA UN CONCEPTO SOCIOLÓGICO DE INNOVACIÓN}

"La innovación solamente se da en sociedad (...), no [irrumpe] en las sociedades desde el más allá, [es] resultado de prácticas y estructuras sociales (...), un producto interactivo" (Innerarity 2009: 30). Además, la innovación es un proceso no solo mercantil, puesto que las mejoras técnicas también implican cambios sociales en las relaciones de poder entre actores. La innovación no es solo destrucción creativa en sentido schumpeteriano sino también reconfiguración social de conocimientos, artefactos y actores (Bender, 2003).

El carácter social de la innovación exige a la Sociología el desarrollo de un utillaje conceptual propio que sea capaz de integrar las aportaciones efectuadas desde otras disciplinas académicas. En este sentido, es posible identificar tres corrientes sociológicas interrelacionadas que esbozan una reflexión teórica sobre la innovación apoyándose en los elementos centrales del paradigma de innovación post-schumpeteriano. La primera de ellas interpreta la innovación como un proceso de creación de valor en sentido marxiano. La segunda efectúa una adaptación de la idea de innovación a las teorías de alcance medio sobre diversidad institucional y cambio social. La tercera propone una teoría dinámica de la organización en la que la innovación se muestra como un elemento operativo esencial.

\section{EL PROCESO DE TRABAJO COMO FUENTE DE INNOVACIÓN}

Desde una perspectiva marxiana, el origen de la innovación se sitúa en el proceso de trabajo. La innovación emerge de la contradicción inherente a la puesta en valor de la fuerza laboral. Dentro del proceso de trabajo, colectivo e interactivo, el trabajador hace uso de su fuerza de trabajo para añadir nuevos elementos al trabajo cosificado, recombinando factores de producción e innovando. La ciencia y la tecnología son formas ideológicas del capital que enmascaran el proceso de cosificación de la actividad innovadora que lleva a cabo la fuerza laboral. En realidad, ni los productos científicos ni los artefactos técnicos generan por si mismos innovación y valor añadido sino que es el trabajo productivo y colectivo el que lo hace posible. El proceso productivo capitalista despolitiza el trabajo humano y difumina sus dimensiones de conflicto y cooperación. Así, cuando una tecnología, sistema de producción o política triunfante se estandariza, se evapora la posibilidad de plantear alguna opción alternativa.

La observación del proceso de trabajo como fuente primaria de innovación permite integrar formas de innovación que se han estudiado separadamente por arenas disciplinares como la gestión del conocimiento, la organización del trabajo o las relaciones laborales. Por ejemplo, la descripción de los modelos de innovación anglosajón y continental europeo como antitéticos (el primero representando la innovación radical de las TICs y el segundo la innovación incremental de los sectores de la industria de nivel tecnológico media) resulta 
operativa en lo descriptivo, pero no cuando se persigue una mayor profundidad analítica.

La contraposición entre modelos de innovación enmascara distintas formas de organizar y gestionar el trabajo. La movilización del trabajo cualificado y su utilización dentro de sistemas de producción heterárquicos, según el modelo estudiado por Carnoy, Castells y Benner (1997) en Silicon Valley, resulta una fuente de innovación rica para determinados sectores y empresas, pero implica graves problemas de generalización. Al mismo tiempo, la implantación de un estilo cooperativo de innovación incremental incrustado en un sistema institucional de innovación y comprometido a largo plazo, según el modelo de capitalismo renano, ofrece una respuesta más lenta a la innovación radical, pero aporta ventajas para el desarrollo inclusivo y sostenido de organizaciones y entornos sociales.

La centralidad de la organización del trabajo y de las relaciones laborales para el análisis de la innovación ha sido abordada por numerosas investigaciones empíricas. Child y Heavens (2001; véase también Lam 2003) destacan la importancia de la organización del trabajo y de las relaciones de poder micropolíticas en la generación de conocimientos a través del aprendizaje informal, en la articulación de redes sociales y en la transferencia de conocimiento dentro y hacia el exterior de la organización. El contrato de trabajo, que facilita la transformación de la fuerza laboral en trabajo efectivo a través de la generación de consentimiento sobre el orden del trabajo, o la motivación del trabajador para cooperar con los objetivos de la organización utilizando y compartiendo los conocimientos prácticos que ha acumulado constituyen fuentes primarias de innovación. La actividad innovadora, como parte de la política del trabajo, implica relaciones de intercambio recíprocas y duraderas, no reducibles a transacciones mercantiles o a la imposición de un determinado orden jerárquico formal en la organización.

\section{INNOVACIÓN, VARIEDAD DE ENTORNOS INSTITUCIONALES Y CAMBIO SOCIAL}

El paradigma post-schumpeteriano de innovación resulta operativo para que la Sociología supere los problemas de polarización entre las teorías de la acción y las teorías sistémicas en la explicación del cambio social. Los procesos de cambio social consolidan lógicas estructurales y senderos de desarrollo, tal y como señalan las teorías sistémicas, pero en ellos debe quedar reflejada la huella de la acción estratégica colectiva e individual.

Los estudios sobre innovación y cambio social identifican cinco momentos de cambio paradigmático en sentido tecno-económico (Deutschmann 2002; Nelson y Winter 1977): (1) la revolución mecánica del vapor aplicada al sector textil (1770-1830); (2) la extensión de esta tecnología a otras áreas de actividad, como el ferrocarril y las manufacturas industriales (1830-1890); (3) el descubrimiento de la electricidad y el desarrollo de la industria pesada (1890- 
1930); (4) la extensión y crisis de la lógica de producción en masa fordista (1930-1980); y (5) la irrupción de la revolución digital y las TICs (1990-¿?). Cada uno de estos cambios de paradigma tecno-económico se acompaña de transformaciones políticas y socio-culturales de amplio alcance.

La investigación sobre la génesis de estos cambios tecnológicos revela la importancia de los modelos de significación colectiva ('Leitbilder') para la consolidación de un sendero de desarrollo tecnológico (Dierkes et al. 1992). En cada momento histórico, los individuos y las organizaciones han desarrollado mapas cognitivos para interpretar su entorno, tomando sobre estos sus decisiones estratégicas sobre los nuevos conocimientos disponibles (Tunzelman y Acha 2003).

Ortmann (1995: 280) explica la dimensión interpretativa y comunicativa de significación de un paradigma tecnológico como el producto de una determinada combinación de modelos de racionalidad, conocimientos y comunicaciones. Se trata de un orden cognitivo y estético desarrollado inicialmente por los técnicos pero del que se apropia la comunidad social, para reproducirse recursivamente a través un conjunto de percepciones e interpretaciones compartidas. Este orden cognitivo y estético facilita la comprensión del nuevo paradigma tecnoeconómico y reduce su incertidumbre, creando nuevas rutinas de cooperación y de comunicación. El desarrollo técnico constituye así un proceso de institucionalización de las tecnologías disponibles dentro de las estructuras sociales, que articulan modos de orientación social y prefiguran futuros senderos de desarrollo tecnológico. El orden institucional limita las opciones de acción social pero también favorece el intercambio de conocimientos tácitos y facilita la puesta en marcha de procesos de innovación incremental. Los efectos retroalimentados entre ventajas competitivas y rutinas consolidan un sendero de desarrollo que combina coherentemente la tecnología y las soluciones organizativas. A cada transición tecno-económica le corresponde una transición socio-cultural. Los ajustes mutuos entre ambas responden a una lógica contingente y conflictiva.

Las aportaciones más recientes a este ámbito de investigación subrayan el carácter abierto de estas transiciones. Los senderos de desarrollo se encuentran condicionados, aunque no determinados, por las revoluciones científicas. La nueva teoría institucional intenta así escapar del carácter estanco de las construcciones teóricas tradicionales sobre diversidad institucional. La existencia de heterogeneidad y contradicción interna es una de las preocupaciones fundamentales de teóricos como Marc Schneiberg (2007). La tensión entre la consolidación de senderos de desarrollo dominantes y sus alternativas emergentes es constante. Un buen ejemplo de ello es la aparente contradicción, dentro de la economía estadounidense, entre el sendero dominante neoliberal y la vitalidad de las iniciativas públicas locales y de las redes de empresas cooperativas.

"Incluso los senderos de desarrollo más asentados se encuentran repletos de desechos flotantes de órdenes industriales alternativos, proyectos abandonados o solo parcialmente realizados y trayectorias no recorridas (...). Estos 
fragmentos (...) son el legado de luchas por el orden cuyas victorias o derrotas contribuyeron a la consolidación del sendero de desarrollo triunfante. Son remanentes de conflictos previos, experimentos fallidos o solo parcialmente exitosos de rutas alternativas y batallas contra aquello que se convirtió en el eje central del orden industrial. Pero, cuando estos legados adquieren suficiente peso, pueden servir para la elaboración de lógicas y trayectorias alternativas" (Schneiberg 2007: 48; traducción propia).

¿Dónde incorporar esta interpretación institucional flexible del vínculo entre cambio social e innovación? Köhler (2007) elabora para ello un concepto analítico a partir de cuatro dimensiones entrelazadas: (1) modelos de racionalidad y visiones orientativas compartidas por los actores ('Leitbild'); (2) discursos de técnicos, que consolidan significados compartidos; (3) políticas de trabajo, orientadas a regular la conflictividad; y (4) dinámicas temporales, que respaldan la consolidación de senderos de desarrollo. La noción de innovación se incorpora a este concepto analítico como integrante de la capacidad de condicionamiento estructural y contingente, pero también como objeto de la acción social reflexiva.

La innovación, en sus dimensiones tecnológica, institucional, política o social, surge del intercambio entre procesos no necesariamente explicables desde la racionalidad de los actores y de sus acciones estratégicas. En ocasiones, los grupos de poder pueden efectuar un elevado esfuerzo para imponer sus tecnologías como estándar, a pesar de que el objetivo de estandarización no se encuentre respaldado por argumentos racionales (Meyer y Schubert 2007).

La innovación no resulta de choques externos o crisis profundas sino que surge a través de la movilización de recursos endógenos alternativos. A medida que los estándares pierden su carácter modélico se incrementan las posibilidades de que una alternativa desechada resurja con fuerza en un nuevo juego político. Con la pérdida de legitimidad de un producto, de un modelo organizativo o de una estrategia de mercado estándar se abren senderos de desarrollo alternativos, a través de un proceso de repolitización y reapertura de corredores de oportunidades cerrados. La heterogeneidad interna de cada sistema institucional es resultado de las posibilidades de isomorfismo (DiMaggio y Powell 1983) y de heteromorfismo de las organizaciones (Lütz 2006).

La importancia de la idea de sistema dentro del paradigma postinstitucionalista de innovación se debe al carácter multi-nivel de la acción innovadora, tanto en un sentido territorial como sectorial. Así, la innovación se convierte en un ítem analítico central de cualquier teoría sociológica del cambio social y de la heterogeneidad institucional.

Al explicar la diversidad institucional y su dinámica, los enfoques de gobernanza inciden en la existencia de redes de intermediación no formales, que operan al margen del mercado a través de lógicas de competencia dispersa, pero también del Estado y sus dispositivos de control jerárquico. Estas redes se entrelazan con la comunidad mediante dispositivos de solidaridad espontánea y asociativa (Cooke y Morgan 1998; Streeck y Schmitter 1985). La innovación constituye una acción asociativa específica que tiene lugar dentro de esta red 
y que es protagonizada por agentes dispersos pero interdependientes, no por individuos racionales aislados o sometidos a un orden jerárquico. Para entender cómo una innovación se extiende e impone como estándar resulta imprescindible estudiar las relaciones de poder multi-nivel que se entrecruzan en el proceso de innovación: desde la micro-política intra-organizacional hasta la geo-política de los sistemas de calidad y certificación.

La conciencia del carácter asociativo de la innovación ha impulsado su aplicación en el ámbito de la gestión pública. El concepto de 'innovación social', que ha sido incorporado a la nueva estrategia comunitaria de innovación, alude a las nuevas ideas, productos, servicios y modelos que atienden simultáneamente a las necesidades sociales y crean nuevas relaciones sociales y colaboraciones (Comisión Europea 2010; 2013). Su objetivo es "revalorizar la fuerte tradición europea de creatividad en áreas como el desarrollo comunitario, el arte o el activismo social, en el desarrollo de una Europa Social Creativa" (Mouleart et al. 2010: 4).

La innovación social es un concepto multidimensional: económico, cultural, social y tecnológico, en el que se entrecruzan diversidad de fuentes y agentes (Echeverría y Gurrutxaga 2012). La existencia de una sociedad civil articulada, con altos niveles de cooperación y participación en los sistemas de gobernanza local, permite buscar soluciones creativas a los problemas de bienestar social. Frente a la innovación organizativo-tecnológica orientada al mercado, la innovación social está motivada "por objetivos que intentan resolver necesidades, problemas y demandas de tipo social y está promovida por organizaciones cuyas principales metas son sociales" (Gurrutxaga 2010: 131).

Por último, un concepto sociológico de innovación debería poner en cuestión la connotación universalmente positiva que recibe la idea de innovación dentro de los discursos de negocio. El cambio social vinculado a la innovación no tiene por qué ofrecer resultados positivos en todos los ámbitos. Por ejemplo, la desregulación de los mercados financieros a escala global asociada a la extensión de innovaciones de gestión financiera, nuevos productos, agentes de regulación o reglas de contabilidad está contribuyendo a configurar un nuevo orden institucional del capitalismo. Este régimen capitalista financializado extiende la presión cortoplacista del espacio financiero al resto de ámbitos relacionales de la organización (Foster 2007). Las formas innovadoras de remuneración que vinculan los ingresos de los gestores al valor bursátil de la empresa han generado un modelo de gobernanza y control corporativo fundamentado en una nueva jerarquía de valores de decisión, normas de gestión y una redistribución de los recursos de poder entre los responsables de la dirección de la empresa. El nuevo orden institucional es innovador, al generar nuevos productos, estilos de gestión y soluciones organizacionales. Pero al mismo tiempo, socava la posibilidad de alcanzar un desarrollo económico social y ecológicamente sostenible (Alonso y Fernández Rodríguez 2012; Kädtler 2010). 


\section{SOCIOLOGÍA DE LAS ORGANIZACIONES E INNOVACIÓN}

Las corrientes de análisis dinámico de la organización que, como el enfoque evolutivo de organizaciones, consideran la capacidad innovadora como un requisito esencial de éxito para las formas organizativas post-burocráticas en su doble perspectiva de exploración de nuevos conocimientos para la organización y de aplicación de los mismos para sus usuarios, se verían también enriquecidas con la incorporación de un concepto sociológico de innovación (Child, 2005; 2012; Montgomery 1995; Pettigrew et at. 2003).

Las empresas desarrollan sus estrategias de beneficio a partir de la combinación de competencias básicas, redes de cooperación con otros actores corporativos, sistemas de producción, productos, valores y normas. La gestión de conocimientos y la innovación ocupan una posición matricial en dichas estrategias de beneficio (Köhler 2008; Boyer y Freyssenet 2003). Pero, nuevamente, la consolidación de una estrategia de beneficio como sendero específico de desarrollo es el resultado de un conjunto de juegos micro-políticos de escaso contenido económico-racional (Münch y Günther 2005).

En un contexto de información limitada e incertidumbre sistémica, la dinámica de las organizaciones tiene sus principales agentes de cambio en las redes de innovación y aprendizaje informal. Estas redes trascienden las fronteras de la organización y facilitan el mutualizado de recursos para la generación de conocimientos compartidos. Pero, al mismo tiempo, son escenario de intrigas, enfrentamientos y alianzas estratégicas de poder (Seely Brown y Duguid 1991).

Los modelos teóricos tradicionales sobre organizaciones resultan incapaces de aprehender estos juegos informales de poder micro-político, sobre los cuales descansan los procesos interactivos de construcción, atesoramiento y difusión de conocimiento e innovación. De ahí la necesidad de elaborar un utillaje teórico que permita la repolitización de los intercambios entre actores como causantes de la institucionalización y desinstitucionalización de las rutinas y prácticas organizativas. Las exigencias de estudio de la innovación no son distintas a las del resto de áreas de expresión de la toma de decisiones corporativas. Como en ellas, la innovación es el resultado contingente de una serie de equilibrios de poder micro-políticos.

\section{CONCLUSIÓN}

El paradigma post-schumpeteriano de innovación, con la sustitución del enfoque de actores racionales por una perspectiva de procesos sociales interactivos y contingentes, introduce una mirada sociológica sobre el análisis de la innovación. Tanto la Economía Evolutiva como el enfoque de sistemas de innovación han elaborado elementos conceptuales perfectamente adaptables a una teoría sociológica de la innovación. El artículo ha detallado las aportaciones potenciales de cada uno de esos elementos conceptuales para el desarrollo de una propuesta teórica específicamente sociológica. 
La teoría marxiana de valor-trabajo ubica la producción de innovación en el interior del proceso de trabajo colectivo. A partir de esta aproximación, se abren nuevas posibilidades para avanzar en la conceptualización del cambio tecnológico como un conjunto complejo de continuidad y transformación no lineal ni necesario y que no resulta en construcciones institucionales monolíticas o poco permeables. La combinación del análisis macro-político de sistemas regionales o sectoriales de innovación con el estudio de los juegos de poder micro-políticos que tienen lugar en las organizaciones proporciona un elemento de reflexión al que es obligatorio referirse en el desarrollo de cualquier concepto sociológico de innovación.

El artículo sugiere un conjunto de direcciones en el proceso de construcción de un concepto sociológico de innovación, recogiendo, ordenando y discutiendo distintas sugerencias teóricas. En este sentido, se es plenamente consciente de que se han dejado al margen algunos problemas prácticos relacionados con la gestión y el fomento público de la innovación (Bender 2006; Lundvall 2002), el análisis de los problemas de sostenibilidad y controlabilidad ecológica de la innovación que propone la Sociología del Riesgo o la ausencia de indicadores apropiados para medir la intensidad de innovación. Son varios los estudios que inciden en el carácter insatisfactorio de los indicadores de innovación más comunes: porcentaje de industrias de alto componente tecnológico, número de patentes, inversión en I+D (Laestadius 2003). Esto es así porque la complejidad social de la innovación rebasa la capacidad operativa que, para su medición, proporcionan los métodos estadísticos, tal y como reconocen los propios organismos oficiales (Comisión Europea, 2013). El desarrollo de una teoría social crítica de la innovación debería contribuir a dar respuesta tanto a estos retos de índole práctica como a aquellas otras preguntas conceptuales a las que el artículo se ha referido con detalle.

\section{BIBLIOGRAFÍA}

ALONSO, L.E. y FERNÁNDEZ RODRÍGUEZ, C.J. (eds.) (2012): La financiarización de las relaciones salariales, Madrid, La Catarata.

BENDER, G. (2003): "Innovation in Low-Tec Companies - Towards a Conceptualisation of Non-Science-Based Innovation", en H. Hirsch-Kreinsen, D. Jacobsen, D. y St. Laestadius (eds.), Low Low-tech Innovation in the Knowledge Economy, Frankfurt. a.M. et al., Peter Lang, pp. 85-98.

BENDER, G. (2006): Peculiarities and Relevance on Non-Research-Intensive Industries in the Knowledge-Based Economy. Final Report of the PILOT project (Policy and Innovation in Low-Tech), University of Dortmund, disponible en http://www.pilotproject.org [consulta 10-5-2013].

BLACKER, F. (1995): "Knowledge, Knowledge Work and Organizations: An Overview and Interpretation", Organization Studies, vol. 16 (6), pp. 1021-1046.

BÖHLE, F. (2010): “Arbeit als Handeln”, en F. Böhle, F., G. Vo囚 y G. Wachtler (eds.): Handbuch Arbeitssoziologie, Wiesbaden, VS Verlag, pp. 151-176. 
BOYER, R. y FREYSSENET, M. (2003): Los modelos productivos, Madrid, Fundamentos.

BRAND, St. (1995): "We Owe It All to Hippies". Time Magazine. (Special Issue: Welcome to Cyberspace).

BUSH, V. (1945): Science The Endless Frontier, A Report to the President by Vannevar Bush, Director of the Office of Scientific Research and Development, United States Government Printing Office, Washington.

CASTRO MARTÍNEZ, E. y FERNÁNDEZ DE LUCIO, I. (2013): El significado de innovar, Madrid, La Catarata.

CHILD, J. (2005): Organization. Contemporary Principles and Practice, Malden et al., Blackwell.

CHILD, J. (ed.) (2012): The evolution of organizations, Cheltenham, Edward Elgar.

CHILD, J. y HEAVENS, S.J. (2001): "The Social Constitution of Organizations and its Implications for Organizational Learning”, en M. Dierkes, M. et al. (eds.), Handbook of Organizational Learning and Knowledge, Oxford: Oxford University Press, pp. 308-326.

COASE, R.H. (1937): "The nature of the firm", ECONOMICA, 16, pp. 386-405.

COHEN, W.M. y LEVINTHAL, D.A. (1990): “Absorptive Capacity: A New Perspective on Learning and Innovation”, Administrative Science Quarterly, 35, pp. 128-152.

COMISIÓN EUROPEA (2010): This is European Social Innovation, Bruselas, Comisión Europea.

COMISIÓN EUROPEA (2013): Unión por la Innovación. Bruselas, Comisión Europea.

COOKE, P., HEIDENREICH, M. y BRACZYK, H.-J. (eds.) (2004): Regional Innovation Systems. The role of governance in a globalized world, London/N.Y ., Routledge, $2^{\text {nd }}$ edition.

COOKE, P. y MORGAN, K. (1998): The Associational Economy, Oxford/N.Y., Oxford University Press.

COOPER, R.G. (2005): Product Leadership, USA: Basic Books.

DENIS, H. (2012): "Marx, Walras y Schumpeter", Revue Interventions économiques, 46/2012.

DEUTSCHMANN, C. (2002): Postindustrielle Industriesoziologie, Weinheim y München: Juventa.

DIERKES, M. (1996): "Sobre paradigmas, aprendizaje y desarrollo empresarial", Situación, 3, pp. 39-55.

DIERKES, M., HOFFMANN, U. y MARZ, L. (eds) (1992): Leitbild und Technik. Zur Entstehung und Steuerung technischer Innovationen, Berlin: Sigma.

DIMAGGIO, P.J. y POWELL, W.W. (1983): "The Iron Cage revisited: Institutional Isomorphism and Collective Rationality in Organizational Fields", American Sociological Review, 48 (2), pp. 147-160.

DOSI, G., TEECE, D.J. y WINTER, S. (1992): “Toward a theory of corporate coherence: Preliminary remarks", en G. Dosi, R. Gianetti y P.A. Toninelli (eds.), Technology and enterprise in a historical perspective, Oxford, Clarendon Press, pp. 185-211.

ECHEVARRÍA, J. y GURRUTXAGA, A. (2012): La luz de la luciérnaga. Diálogos de Innovación Social, Madrid: Plaza y Valdés.

ELLIOT, J.E. (1980): “Marx and Schumpeter on Capitalism's Creative Destruction: A Comparative Restatement", The Quarterly Journal of Economics, 95, August 1980, pp. 45-68. 
EVELEENS, C. (2010): "Innovation management; a literature review of innovation process models and their implications", disponible en http://www.lectoraatinnovatie. $\mathrm{nl} / \mathrm{wp}$-content/uploads/2011/01/Innovation-management-literature-review-.pdf [consulta 10-5-2014].

FOSTER, J.B. (2007): “The Financialization of Capitalism”, Monthly Review, 58 (11), pp. $1-12$.

FREEMAN, C. (1987): Technology Policy and Economic Performance: Lessons from Japan, London, Pinter.

FREEMAN, C. (2002): "Continental, national and sub-national innovation systems complementarity and economic growth", Research Policy, 31, pp. 191-211.

GURRUTXAGA, A. (2010): Recorridos por el cambio, la innovación y la incertidumbre, Bilbao: Universidad del País Vasco.

HALL, P.A. y SOSKICE, D. (2001): "An Introduction to Varieties of Capitalism. Varieties of Capitalism: The Institutional Foundations of Comparative Advantage", en P.A. Hall y D. Soskice (eds), Varieties of Capitalism: The Institutional Foundations of Comparative Advantage, Oxford, Oxford University Press, pp. 1-68.

HAYEK, F.A. (1945): "The use of knowledge in society", The American Economic Review, 35 (4), pp. 519-530.

HEIDENREICH, M. (2004): "Conclusion. The dilemmas of regional innovation systems", en P.N. Cooke, M. Heindenreich y H.J. Braczyk (eds.), Regional Innovation Systems. The Role of Governance in a Globalized World, London: Routledge, pp.363-389.

HENDERSON, R.M. y CLARK, K.B. (1990): “Architectural Innovation: The Reconfiguration of Existing Product Technologies and the Failure of Established Firms", Administrative Science Quarterly, 35, pp. 9-30.

HIRSCH-KREINSEN, H. (2002): "Knowledge in Societal Development: The Case of Low-Tech Industries “, Universität Dortmund, Arbeitspapiere des Lehrstuhls Technik und Gesellschaft.

INNERARITY, D. (2009): "La sociedad de la innovación”, en Innobasque (comp..), ¿Cómo es una sociedad innovadora?, Zamudio, Innobasque, pp. 18-41, disponible en http://www.innobasque.com/home.aspx?tabid=762 [consulta 13-5-2013].

JÜRGENS, U. y SABLOWSKI, T. (2005): "Die Vielfalt sektoraler Innovationsprozesse - Pharmaindustrie, Telekommunikation, Autoindustrie“, WSI-Mitteilungen, 58 (3), pp. 121-129.

KÄDTLER, J. (2010): “Finanzmärkte und Finanzialisierung”, en F. Böhle, F., G. Vo® y G. Wachtler (eds.), Handbuch Arbeitssoziologie, Wiesbaden: VS Verlag, pp. 619639.

KÖHLER, H.-D. (2007): "Warum arbeiten wir immer noch am Fliessband? Zum QWERTY-Effekt in Produktionssystemen”, Zeitschrift für Arbeitswissenschaft, 61 (1), pp. 59-66.

KÖHLER, H.-D. (2008): "Profit and innovation strategies in low-tech firms", Estudios de Economía Aplicada, 26 (3), pp. 73-87.

LAESTADIUS, St. (2003): "The classification and taxonomy of industries - Measuring the right thing", en H. Hirsch-Kreinsen, D. Jacobsen, D. y St. Laestadius (eds.), Low-tech Innovation in the Knowledge Economy, Frankfurt. A.M. et al.: Peter Lang, pp. 63-84.

LAM, A. (2000): "Tacit knowledge, organizational learning and societal institutions: An integrated framework", Organization Studies, 21 (3), pp. 487-513.

LAM, A. (2003): "Skills and careers of $R \& D$ Knowledge Workers in the Netw 
LAM, A. (2003): "Skills and careers of $R \& D$ Knowledge Workers in the Network Economy", Paper presented at the IIRA $13^{\text {th }}$ World Congress, Berlin.

LARREA, J.L. (2010): “Paradojas en la Innovación”, San Sebastián: Orkestra Working Paper No 2010-DO1.

LOASBY, B.J. (2002): "The evolution of knowledge: beyond the biological model", Research Policy, 31, pp. 1227-1239.

LUNDVALL, B.A. (2002): Innovation, growth and social cohesion. The Danish model, UK/Northampton, USA, Cheltenham.

LUNDVALL, B.Å., JOHNSON, B., ANDERSONEN, E.S. y DALUM, B. (2002): "National systems of production, innovation and competence building", Research Policy, 31, pp. 213-231.

LÜTZ, S. (2006): "Zwischen Pfadabhängigkeit und Wandel. ,Governance“ und die Analyse kapitalistischer Institutionenentwicklung", en U. Brinkmann, K. Krenn y S. Schief (eds.), Endspiel des kooperativen Kapitalismus?, Wiesbaden: VS Verlag, pp. 16-34.

MACKENZIE, D. (1984): "Marx and the Machine", Technology and Culture, 25 (3), pp. 473-502.

MALERBA, F. (ed.) (2004): Sectoral Systems of Innovation, Cambridge Mass., Cambridge University Press.

MARX, K. (1946, 1867): El Capital, México: FCE.

MCKINLAY, A. (2005): "Knowledge Management", en St. Ackroyd, R. Batt, P. Thompson y P.S. Tolbert (eds.): The Oxford Handbook of Work and Organization, Oxford/N.Y., Oxford University Press, pp. 242-262.

MEYER, J.W. y ROWAN, B. (1977): "Institutionalized Organizations: Formal Structure as Myth and Ceremony", American Journal of Sociology, 83 (2), pp. 340-363.

MEYER, U. y SCHUBERT, C. (2007): "Integrating path dependency and path creation in a general understanding of path constitution. The role of agency and institutions in the stabilization of technological innovations", Science, Technology \& Innovation Studies, 3, pp. 23-44.

MONTGOMERY, C.A. (ed.) (1995): Resource-Based and Evolutionary Theories of the Firm: Towards a Synthesis, Boston et al., Kluwer.

MOULAERT, F., MACCALLUM, D., MEHMOOD, A. y HAMDOUCH, A. (eds.) (2010): Social Innovation: Collective action, social learning and transdisciplinary research, Bruselas: Unión Europea, disponible en http://cordis.europa.eu/documents/ documentlibrary/124376771EN6.pdf [consulta 13-5-2013].

MÜNCH, R. y GUENTHER, T. (2005): "Der Markt in der Organisation. Von der Hegemonie der Fachspezialisten zur Hegemonie des Finanzmanagements", en P. Windolf (ed.) (2005): Finanzmarkt-Kapitalismus, Sonderheft 45 der KZfSS, pp. 394-417.

MYTELKA, L.K. y SMITH, K. (2002): "Policy learning and innovation theory: An interactive and co-evolving process", Research Policy, 31: 1467-1479.

NELSON, R., (ed.) 1993: National Innovation Systems. A Comparative Analysis, Oxford: Oxford University Press.

NELSON, R. y WINTER, S. (1977: "In search of useful theory of innovation", Research Policy, 6, pp. 36-76.

NONAKA, I. (1994): “A Dynamic Theory of Organizational Knowledge Creation”, Organization Science, 5 (1), pp. 14-37.

ORTMANN, G. (1995): Formen der Produktion, Opladen: Westdeutscher Verlag. 
PAVITT, K. (1984): "Sectoral patterns of technical change: towards a taxonomy and a theory", Research Policy, 13 (6), pp. 343-373.

PAVITT, K. (1992): "Some foundations for a theory of the large innovating firm", en G. Dosi, R. Gianetti, P.A. Toninelli (eds.), Technology and enterprise in a historical perspective, Oxford: Clarendon Press, pp. 212-228.

PENROSE, E. (1959): The Theory of the Growth of the Firm. Basil Blackwell, Oxford.

PETTIGREW, A.M. (2003): Innovative forms of organizing, London/Thousand Oaks/ New Delhi, Sage.

POLANYI, M. (1966): The tacit dimension, N.Y., Garden City.

PORTER, M. (1990): The competitive advantages of Nations, London, MacMillan.

PORTER, M. (1998): "Clusters and the new economies of competition", Harvard Business Review, 76 (6), 77-90.

ROGERS, E.M. (1995, 1962): Diffusion of innovation, New York, The Free Press $4^{\text {th }}$ ed.

ROSENBERG, N. (1993): Dentro de la caja negra: tecnología y economía, Barcelona, La Llar del Llibre.

SCHNEIBERG, M. (2007): "What's on the path? Path dependence, organizational diversity and the problem of institutional change in the US economy, 1900-1950", Socio-Economic Review, 5, pp. 47-80.

SCHUMPETER, J.A. (1911, 1957): Teoría del desenvolvimiento económico: una investigación sobre ganancias, capital, crédito, interés y ciclo económico, México, FCE

SCHUMPETER, J.A. (1942, 1971): Capitalismo, Socialismo y Democracia, Madrid, Aguilar.

SEELY BROWN, J. y DUGUID, P. (1991): "Organizational learning and communitiesof-practice", Organization Science, 2, pp. 40-57.

SERRA RAMONEDA, A. (2001): "Empresa, información y/o conocimiento", en M.A. Durán et al. (eds.), Estructura y cambio social. Homenaje a Salustiano del Campo, Madrid, CIS, pp. 791-808.

SPENDER, J.C. (1996): "Making Knowledge the Basis of a Dynamic Theory of the Firm", Strategic Management Journal, vol. 17, 45-62.

STREECK, W. y SCHMITTER, P. (1985): "Community, market, state - and associations? The prospective contribution of interest governance to social order", en W. Streeck y P. Schmitter (eds.), Private Interest Government, London et al., Sage, pp. 1-29.

TEECE, D.J., PISANO, G y SHUEN, A. (1997): "Dynamic capabilities and strategic management", Strategic Management Journal, 18 (7), pp. 509-533.

TUNZELMANN, N. y ACHA V. (2003): Innovation in 'Low-Tech' Industries. University of Sussex, TEARI working paper $\mathrm{n}^{\circ} 15$.

WERLE, R. (2005): "Institutionelle Analyse technischer Innovation", Kölner Zeitschrift für Soziologie und Sozialforschung, 57 (2), pp. 308-332.

WHITLEY, R. (1999): Divergent Capitalisms. The Social Structuring and Change of Business Systems, Oxford, Oxford University Press.

WILLIAMSON, O.E. (1981): "The Economics of Organization: The Transaction Cost Approach", American Journal of Sociology, 87 (3), pp. 548-577.

WILLIAMSON, O.E. (1985): The Economic Institutions of Capitalism. Firms, Markets, Relational Contracting, New York, Free Press. 